\begin{tabular}{ccc}
\hline & $\begin{array}{c}\text { International Journal of Health Services } \\
\text { Research and Policy } \\
\text { www.dergipark.org.tr/ijhsrp }\end{array}$ \\
INTESEG & e-ISSN: 2602-3482 \\
ENGINEERING \\
SCIENCE AND \\
EDUCATION GROUP
\end{tabular}

Research Article

\title{
EVALUATION OF CLINICAL USE HABITS OF TUMOR MARKER TESTS
}

\author{
Ali Volkan OZDEMIR*1 (iD Soycan MIZRAK ${ }^{2}$ (D) \\ ${ }^{1}$ Usak Research and Training Hospital, Clinical Biochemistry, TURKEY \\ ${ }^{2}$ Usak University Faculty of Medicine, Clinical Biochemistry, TURKEY \\ "Corresponding author; dralivolkan@gmail.com
}

\begin{abstract}
Tumor markers (TMs) result from the re-expression of substances by embryologically related tissues. Many are found in different tumors of the same tissue. Therefore, they have low specificity and are not sufficiently sensitive as a screening test. The aim of this study is to evaluate the TM requesting habits of clinicians in Usak Training and Research Hospital, and the appropriateness of the test requests with the diagnosis. Data of 6998 serum TMs requested from 6531 patients between May 1 and July 31, 2019, were obtained from Laboratory Information System and grouped as sex, age, disease diagnoses, and multiple requests (more than 3 tests simultaneously). Compliance with the diagnosis was evaluated as appropriate or inappropriate based on published guidelines for indications for TM requests. Most TMs were made in the 50-70 age range (48.3\%). Multiple TMs were mostly demanded from the Obstetrics and Gynecology Clinic with the diagnosis of menstrual irregularity. Also, 1078 of 1408 total PSA and 28 of 191 free PSA tests were requested with appropriate pre-diagnosis. This study is an example of the use of data mining for the conformity assessment purposes of the TM requests. Accordingly, it was found that the TMs were often incompatible with the diagnosis and were used for general screening purposes. In order to minimize misuse, evidence-based indicators should be developed and clinician awareness should be increased by creating test request algorithms that support the diagnosis.
\end{abstract}

Keywords: Tumor marker, test request, appropriateness

Received: February 22, $2020 \quad$ Accepted: June 18, 2020

\section{Introduction}

The disease that leads to deterioration of body functions by the uncontrolled growth, proliferation, or spread of a cell to surrounding tissues is called cancer. Cancer is a public health problem, and it is the leading cause of death worldwide. Each year, the costs of cancer diagnosis, treatment, and care constitute an important part of the national economic burden [1]. Tumor markers can use for diagnosis of cancer disease, determination of prognosis, monitoring of the treatment process. These markers can analyze from blood, urine, feces, and body fluids [2].

An ideal tumor marker should [3]; (a) be highly specific to a given tumor type; (b) provide a lead-time over clinical diagnosis; (c) be highly sensitive to avoid false-positive results. Additionally, (d) the levels of the marker should correlate reliably with the tumor mass, accurately reflecting any tumor progression or regression, along with a short half-life allowing frequent serial measurements. (e) The 
test used for detection should be cheap for screening applications at the mass level and should be of such nature as to be acceptable to the target population. (f) It should not be detected healthy individual's body and in benign conditions or measured at much lower levels than in cancer cases. In reality, an ideal tumor marker does not exist according to this.

Tumor markers (TMs) result from the re-expression of substances by embryologically related tissues. Many found in different tumors of the same tissue [4]. Therefore, they have low specificity and are not sufficiently sensitive as a screening test except for PSA. Although markers other than PSA have no place in routine screening, they frequently used in clinical settings with incorrect indications. In this study, we intended to evaluate the request of tumor marker tests at Uşak Training and Research Hospital according to age, gender, and pre-diagnosis.

\section{Materials and Methods}

Tumor marker test results including carcinoembryonic antigen (CEA), alfa-fetoprotein (AFP), thyroglobulin (TG), carbohydrate antigen(CA) 125, CA15.3, CA19.9, prostate-specific antigen (PSA), free prostate-specific antigen (fPSA) from patients aged 18 years and over were obtained from Laboratory Information System between 1 May and 31 July 2019 at Uşak University Training and Research Hospital. Obtained data were evaluated by taking into consideration criteria such as age groups, gender, outpatient- clinic requests, appropriateness of prediagnosis, normal-pathologic values, and group marker tumor demand. Three or more test requests in one examination of a patient were evaluated as "Group Test Request". The request for free PSA (fPSA) test without a previously studied PSA test was called "Inappropriate fPSA Request". Thyroglobulin test was performed on Immulite 2000 (Siemens Healthineers USA) and all other TM tests were performed on ADVIA Centaur XP Immunoassay (Siemens, Munich, Germany) autoanalyzer. All internal quality control (BIO-RAD, Lypocheck Tumor Marker Plus, United States) and external quality control (BIO-RAD, EQAS, QCnet international) analysis performed at the time of the period that the data collected were in acceptable ranges. The values indicated by the manufacturers in the kit package insert were accepted as the reference ranges for the tumor marker tests. Microsoft EXCEL and SPSS 21.0 programs used for the classification and statistical analysis of the frequency of test requests and calculate the percentage ratios. The distribution of the tests requested according to the clinics and diagnosis were examined. The distribution of the group test requests according to the clinics was examined. In addition, the request patterns of PSA and PPSA tests were examined.

The examined clinical scenario was the use of TMs in the general practice in which a meaningful overprescription has shown. The performance indicators developed considering both available evidence and implementation feasibility [5]. Previously defined evidence-based criteria were followed to develop performance indicators [6].

The performance indicators developed in the present study reported as below:

1. Age: According to the Clinical Practice Guide (CPG), the eligibility of requests from persons under the age of 40 is discussed. In accordance with this criterion, the cases under the age of 40 were checked.

2. Gender: According to CPG, TM (PSA, CA125, CA 15,3) is sex-specific due to some malignancies. In this study, we specifically evaluated whether these TM requests were limited to gender in which malignancy was common. 
3. Diagnosis: According to CPG, many tumor marker tests are recommended for the follow-up of patients diagnosed with malignancy. In this study, it was evaluated how much of the TM requests were demanded from patients with a diagnosis or a pre-diagnosis of malignancy.

4. Group Test Requests: Since other tumor markers other than PSA were non-tissue specific, 3 or more TM requests at the same time were considered inappropriate.

Ethics Committee's Name: Local ethical committee of Usak University Faculty of Medicine

Ethics Committee approval number and date: 17 / 22.11.2019

\section{Results}

Of the $6998 \mathrm{TM}$ tests, 6202 were requested from outpatients and 796 from inpatients. The distribution of test requests according to gender was determined as 4168 females and 2830 males. The distribution of tumor marker tests performed is shown in Table-1. Accordingly, the most demanded tests were CEA and PSA.

Table 1. TM Tests Distribution

\begin{tabular}{|l|c|c|}
\hline \multicolumn{1}{|c|}{ Tests } & Frequency & Percent \\
\hline CA 125 & 1027 & 14.7 \\
CEA & 1521 & 21.7 \\
CA 19-9 & 1163 & 16.6 \\
fPSA & 191 & 2.7 \\
CA 15-3 & 965 & 13.8 \\
PSA & 1408 & 20.1 \\
AFP & 615 & 8.8 \\
TG & 108 & 1.5 \\
Total & 6998 & 100.0 \\
\hline
\end{tabular}

The distribution of the tests according to the clinics and the rate of requesting appropriate diagnosis are given in Table-2. The ratio of TM tests requested per patient is quite similar among all TM tests.

Table 2. Test distribution according to clinics, the ratio of requests with appropriate diagnosis

\begin{tabular}{|c|c|c|c|c|c|c|c|c|c|}
\hline Tests & AFP & CA 15-3 & CA 19-9 & CA 125 & CEA & fPSA & PSA & TG & Total \\
\hline İnternal Medicine (n) & 316 & 148 & 233 & 198 & 225 & 101 & 206 & 15 & 1442 \\
\hline Gastroenterology (n) & 98 & 3 & 12 & 0 & 14 & 0 & 0 & 0 & 127 \\
\hline Surgeries (n) & 51 & 198 & 257 & 187 & 290 & 37 & 1132 & 8 & 2160 \\
\hline Endocrinology (n) & 6 & 2 & 0 & 0 & 0 & 0 & 11 & 83 & 102 \\
\hline Oncology (n) & 23 & 274 & 295 & 101 & 644 & 53 & 59 & 1 & 1450 \\
\hline Gynecology (n) & 121 & 340 & 365 & 541 & 349 & 0 & 0 & 1 & 1717 \\
\hline Total (n) & 615 & 965 & 1162 & 1027 & 1522 & 191 & 1408 & 108 & 6998 \\
\hline $\begin{array}{c}\text { With appropriate pre-diagnosis(n) } \\
\text { The ratio of appropriate pre-diagnosis } \\
\text { to the number of patients (\%) }\end{array}$ & $\mathbf{4 8}$ & $\mathbf{1 3 . 7 0}$ & $\mathbf{2 8}$ & $\mathbf{6 1}$ & $\mathbf{4 0}$ & $\mathbf{1 4 . 6 0}$ & $\mathbf{7 6 . 5 0}$ & $\mathbf{8 6}$ & $\mathbf{4 5 . 5 0}$ \\
\hline Number of patients (n) & 608 & 847 & 1007 & 1015 & 1368 & 183 & 1397 & 106 & 6531 \\
\hline Avg test per patient & 1.01 & 1.13 & 1.15 & 1.01 & 1.11 & 1.04 & 1.00 & 1.01 & 1.1 \\
\hline
\end{tabular}


Table 3. Diagnoses recorded for test requested

\begin{tabular}{|l|l|l|}
\hline DIAGNOSES & Frequency & $\%$ \\
\hline No diagnosis, General examination & 1878 & 26.8 \\
\hline Non-malignant gynecological diagnoses & 889 & 12.8 \\
\hline Benign prostatic hyperplasia & 733 & 10.5 \\
\hline Pain & 671 & 9.7 \\
\hline Menstrual disorders & 498 & 7.2 \\
\hline Vitamin deficiencies & 413 & 5.9 \\
\hline Other diagnoses not associated with malignancy & 477 & 6.9 \\
\hline Non-malignant urologic diagnoses & 378 & 5.4 \\
\hline Non-malignant gastroenterological diagnoses & 278 & 4.0 \\
\hline Viral Hepatitis & 193 & 2.8 \\
\hline Other disorders of the breast & 181 & 2.6 \\
\hline Non-malignant thyroid disorders & 131 & 1.9 \\
\hline Other liver diseases (including cirrhosis and fibrosis) & 57 & 0.8 \\
\hline Malignancy related other diagnoses (Colon, Breast, Lung, Kidney) & 52 & 0.7 \\
\hline Malignant prostate neoplasm & 44 & 0.6 \\
\hline Unspecified masses in any part of the body & 30 & 0.4 \\
\hline Unspecified mass in the breast & 30 & 0.4 \\
\hline Abnormal weight loss & 22 & 0.3 \\
\hline Total & 6998 & \\
\hline
\end{tabular}

Table-3 shows the distribution of diagnoses entered when requesting tumor marker assays. Accordingly, $26.8 \%$ of the tests were requested from patients without any diagnosis. The most commonly used diagnoses were non-malignant gynecological diseases (12.8\%).

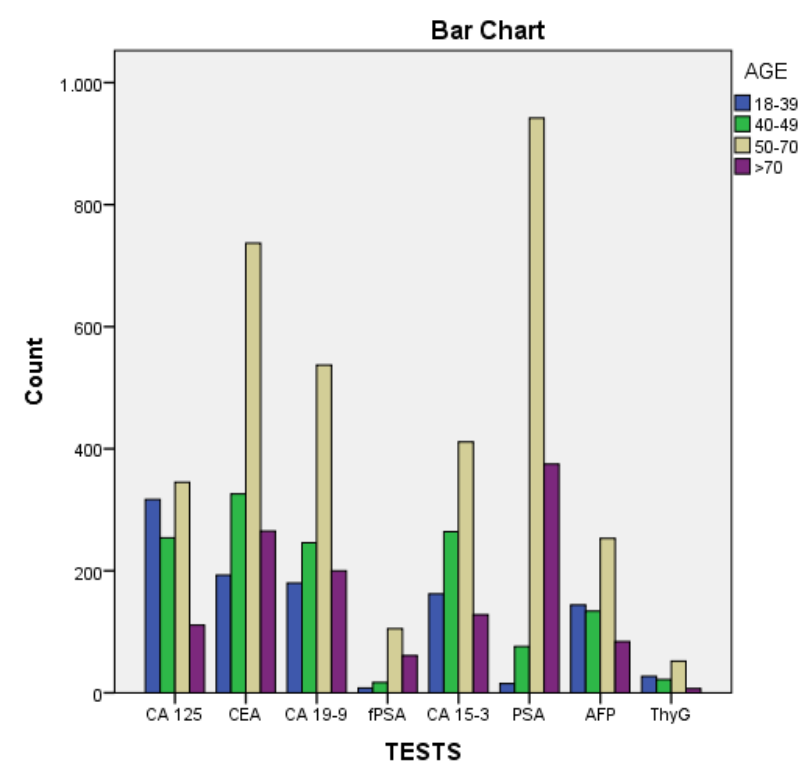

Figure 1. Distribution of Tests According to Age Groups 
The age range where tumor marker tests were most frequently requested was found to be 50 $70(48.3 \%)$. In this study, the rate of TM requests under the age of 40 was found to be $15 \%$. It is noteworthy that $30.8 \%$ of the CA 125 test requests were made from patients aged 18-39 years (Figure1).

When the group requests are evaluated, it is seen that glycoprotein type TM tests (CA125-CA 15,3CA 19,9-CEA) are frequently requested together. $\% 46.3$ of group test requests were ordered from the gynecology clinics (Table 4). Accordingly, the most commonly recorded diagnoses in the group test requests are those related to gynecological diseases (Menstrual disorders, etc ).

Table 4. Clinical Distribution of Group Requests

\begin{tabular}{|l|c|c|}
\hline CLINICS & Frequency & \% \\
\hline Gynecology & 340 & 46.3 \\
\hline Internal Medicine & 184 & 25.1 \\
\hline Oncology & 98 & 13.4 \\
\hline Surgery & 112 & 15.3 \\
\hline Total & 734 & 100.0 \\
\hline
\end{tabular}

The fPSA and PSA claims were further evaluated. Accordingly, fPSA requests are grouped as "Single appropriate fPSA Requests without PSA", Inappropriate fPSA requests with tPSA ", "Single appropriate fPSA "“ Appropriate fPSA Requests with PSA ”. Only 37.7\% of the fPSA tests were considered as appropriate requests (Table-5).

Table 5. Request Compatibility of fPSA

\begin{tabular}{|l|c|c|}
\hline & Frequency & \% \\
\hline Single fPSA Requests without tPSA & 42 & 22.0 \\
\hline Inappropriate fPSA requests with tPSA & 71 & 37.2 \\
\hline Single appropriate fPSA Requests without tPSA & 6 & 3.1 \\
\hline Appropriate fPSA Requests with tPSA & 72 & 37.7 \\
\hline Total & 191 & 100.0 \\
\hline
\end{tabular}

\section{Discussion}

Tumor markers are minimally invasive, low-cost, and easily accessible tests that are used primarily for determining disease prognosis and treatment planning. It is important to use such easily accessible tests at the right time and to interpret them correctly. Guidelines to assist clinicians in this matter should put forward more effectively. Appendix 1 shows the diagnosis of the criteria of TM test requests. Laboratory specialists should consider these criteria when determining test request rules and arranging request papers. 
Appendix 1: Appropriate TM Requests [7]

\begin{tabular}{|c|c|}
\hline TUMOR & DIAGNOSIS CRITERIA OF TM TEST REQUESTS \\
\hline AFP & $\begin{array}{l}\text { 1. Independent prognostic marker for NSGCT (e.g. of the testis). } \\
\text { 2. Diagnostic aid for hepatocellular carcinoma and hepatoblastoma } \\
\text { 3. Screening for hepatocellular carcinoma in high-risk populations }\end{array}$ \\
\hline Ca 15.3 & $\begin{array}{l}\text { 1. Detecting recurrences in asymptomatic patients with diagnosed breast cancer. } \\
\text { 2. For monitoring the treatment of patients with advanced breast cancer }\end{array}$ \\
\hline CA 19.9 , & Monitoring treatment of patients with pancreatic adenocarcinoma \\
\hline CA 125 & $\begin{array}{l}\text { 1. it is helpful for monitoring treatment with chemotherapy in ovarian cancer disease. } \\
\text { 2. } \quad \text { its measurement in postmenopausal patients with pelvic masses may help } \\
\text { differentiate malignant from benign lesions }\end{array}$ \\
\hline CEA & $\begin{array}{l}\text { Helpful in surveillance following curative resection of colorectal cancer and in } \\
\text { monitoring therapy in advanced colorectal cancer. }\end{array}$ \\
\hline PSA & $\begin{array}{l}\text { Diagnosis of prostate cancer and useful in determining prognosis, surveillance } \\
\text { following diagnosis, and monitoring therapy in patients with prostate cancer. }\end{array}$ \\
\hline TG & TG is useful in monitoring the thyroid malignancies. \\
\hline fPSA & Total PSA values are between 4 and $10 \mu \mathrm{g} / \mathrm{L}$ \\
\hline
\end{tabular}

In this study, approximately 55\% of TM orders were found to be incompatible with the diagnosis. PSA tests constitute $76.5 \%$ of the diagnostic-compliant TM orders. When we evaluated the diagnoses from patient records, we saw that almost $30 \%$ of the requested tests were with the diagnosis of general examination or without any pre-diagnosis. This suggests that physicians use TM tests as a routine biochemistry analysis. Deasy K et al showed that $79 \%$ of the orders were inappropriate [8]. The inappropriate use of TM tests could be harmful to the patient-clinician and government economy. Unnecessary TM tests will bring psychological stress to the patient as well as unnecessary further examination.

Different methods tried to prevent unnecessary test requests. For example, Durand et al. reported that due to small changes in test request forms, they reduced the inappropriate test request. In the forms, they wrote the organs where tumor markers were most affected and performed the tests on the prompt screen by separating the sections rather than one after the other [9]. Also, Ferraro et al checked all requests containing more than two TMs. Several of those were performed for diagnostic purposes. The most frequent and inappropriately requested TMs were carcinoembryonic antigen and carbohydrate antigen 19.9 [10]. In our study, group requests were made with glycoprotein structure tests (CA19.9, CA 15.3, CA125). Although these tests are structurally similar, it should be kept in mind that the organ pathologies to which they are primarily affected are different. The majority of these group test request is pelvic inflammatory disease (PID), ovarian cysts, and endometriosis.

Malignancy related diagnoses are only $1.3 \%$ of the total. Tumor marker requests from patients 
with nonspecific diagnoses such as pain, menstrual disorders, and vitamin deficiencies are quite high, indicating that these tests are used for routine screening and / or are not deliberately requested. In conclusion, if the frequency of inappropriate tests decreases, environmental, economical, unnecessary loss of workforce will be prevented, patients' waiting times will be shortened and contributions will be made to eliminate many problems including patient psychology.

Ethics Committee's Name: Local ethical committee of Usak University Faculty of Medicine

Ethics Committee approval number and date: 17 / 22.11.2019

The compliance to Research and Publication Ethics: This work was carried out by obeying research and ethics rules.

\section{References}

[1] NIH, What is cancer, NIH (National Institutes of Health-National Cancer Institute), www.cancer.gov/cancertopics/cancerlibrary/what-is-cancer, 2012.

[2] Sharma S,. "Tumor markers in clinical practice: General principles and guidelines", Indian J Med Paediatr Oncol., 30(1), 1-8, 2009.

[3] Sturgeon C. "Practice guidelines for tumor marker use in the clinic", Clin Chem., 48(8), 1151-9, 2002.

[4] Liu J., "The dualistic origin of human tumors”, Semin Cancer Biol., 53, 1-16, 2018.

[5] Gion M et al., "An epidemiology-based model as a tool to monitör the outbreak of inappropriateness in tumor marker requests: a national scale study", Clin Chem Lab Med., 54(3), 473-482, 2016.

[6] Gion $\mathrm{M}$ et al., "Indicators of inappropriate tumor marker use through the mining of electronic health records", J Eval Clin Pract., 23(7), 1-8, 2017.

[7] Scientific Committee of the Association of Clinical Biochemists in Ireland (ACBI), The Association of Biochemists in Ireland - Guidelines for the use of tumor markers, Fourth edition: Clinical Biochemistry field, Ireland, 2010.

[8] Deasy K et al., “Are We Ordering Tumor Markers Appropriately?” American Journal of Medicine and Medical Sciences, 7(3), 151-155, 2017.

[9] Durand-Zaleski I et al., "Reducing Unnecessary Laboratory Use with New Test Request Form: Example of Tumor Markers", The Lancet, 342, 150-153, 1993.

[10] Ferraro S et al., "Tumor Marker Ordering: Do Not Lose Control: A Prospective Clinical Trial”. American Journal of Clinical Pathology, 144 (4), 649-658, 2015. 